\title{
ATUAÇÃO DO FARMACÊUTICO NA FARMÁCIA POPULAR NO MUNICÍPIO DE NOVA IGUAÇU
}

\author{
Elaine Venâncio Alves da Silva ${ }^{1}$ \\ Leonardo Guimarães de Andrade 2
}

RESUMO: No nosso país, o acesso à saúde é um conceito constitucional zelado pelo Estado, com princípios que devem ser universais, integral, igualitário e com controle social. Portanto, deve-se ser compreendida pela política pública que gerencie as ações para que assim o acesso aos medicamentos essenciais, de forma qualificada e racional, sejam distribuídas de forma estratégica. Para que todo esse processo funcione de forma ampla, o trabalho do farmacêutico nas farmácias populares é de extrema importância. Neste trabalho vamos ver o quanto esse serviço é fundamental no município de Nova Iguaçu, orientação aos pacientes sobre as dúvidas relacionadas à saúde, preocupações com doenças, sintomas, riscos e auxílio na prevenção de futuras doenças, iniciativas voltadas para o bem-estar, proteção, segurança e recuperação dos mesmos.

Palavra-chave: Atuação do farmacêutico. Farmácia popular no município de Nova Iguaçu.

ABSTRACT: In our country, access to health is a constitutional concept zealous by the State, with principles that must be universal, integral, egalitarian and with social control.Therefore, it should be understood by public policy that manages actions so that access to essential medicines, in a qualified and rational way, is distributed strategically. For this whole process to work broadly, the work of the pharmacist in popular pharmacies is extremely important.In this work we will see how fundamental this service is in the city of Nova Iguaçu, guidance to patients on health-related doubts, concerns about diseases, symptoms, risks and assistance in the prevention of future diseases, initiatives aimed at their well-being, protection, safety and recovery.

Keyword: Performance of the pharmacist. Popular pharmacy in the municipality of Nova Iguaçu.

\section{OBJETIVO GERAL}

No município de Nova Iguaçu, o programa farmácia popular foi implantada em 2017, porém já era conhecida em 2004, quando foi implantada pelo Ministério da Saúde. Nos dias de hoje tem parcerias com farmácias privadas. O objetivo desta

\footnotetext{
r Universidade Iguaçu. nane.venancio@hotmail.com.br

${ }^{2}$ Orientador. Universidade Iguaçu.
} 
pesquisa é avaliar a relação paciente e farmacêutico, considerando as diversas barreiras para o aperfeiçoamento de acesso a medicamentos.

\section{OBJETIVOS ESPECÍFICOS}

- Compreender o programa farmácia popular no município de Nova Iguaçu;

- Analisar o acesso e dificuldades aos medicamentos;

- Verificar a relação que o farmacêutico exerce ao público em informações;

- Orientações e benefícios, com base nas atuais situações da Política Nacional de Medicamentos;

- Compreender a Política Nacional de Assistência Farmacêutica, especialmente no quesito fortalecimento de medicamentos genéricos;

\section{METODOLOGIA}

O programa de Farmácia Popular no município de Nova Iguaçu tem em vista um projeto de renda indireta condicional ao uso de medicamentos específicos. Baseado nisto, o governo realiza os gastos de implantação da demanda dos devidos medicamentos para serem disponibilizados para os cidadãos. Assim, no ato da compra o beneficiado percebe a redução de preços dado através do copagemento, observando um aumento na renda disponível para o consumo paralelo. Os medicamentos oferecidos são definidos pelo programa para doenças crônicas e com uso obrigatório e contínuo. A quantidade estabelecida é definida no receituário, não podendo haver alterações. Portanto, especificamente, em Nova Iguaçu é de extrema importância esse programa, para que os pacientes se sintam seguros e acolhidos com preços justos. Esse estudo sendo abordo no período de 2017 a 2021.

\section{JUSTIFICATIVA}

Tendo sido criado essa pesquisa, abordei a profissão do farmacêutico e o direito do cidadão sobre os medicamentos oferecidos pelo programa, sem esquecer da importância das farmácias e drogarias que fazem parte deste projeto. Pesquisas apontam a efetivação da integridade na saúde em Nova Iguaçu junto com o projeto, 
que antigamente enfrentavam dificuldades em relação a orientação e em obter medicamentos específicos.

\section{DESENVOLVIMENTO}

Criado e regulamentado em 2004 pela Lei $\mathrm{n}^{0}$ 10.858 e Decreto $\mathrm{n}^{\mathrm{o}}$ 5.090, o Programa Farmácia Popular do Brasil tem como propósito atender à Política Nacional de Assistência Farmacêutica, amplificando o acesso a remédios considerados fundamentais para tratamentos específicos. Logo após o fechamento dos estabelecimentos próprios em 2017, passou a funcionar somente em farmácias e drogarias comerciais credenciadas, beneficiando a cadeia farmacêutica. Em 2007, após publicação da Portaria no I.4I4, foram incluídos os anticoncepcionais. (KORNIS; BRAGA; ZAIRE, 2008).

Três anos depois, incluíram a insulina regular e a sinvastatina, para combater o colesterol alto. No mês de outubro do mesmo ano, o programa incluiu os medicamentos para o tratamento de osteoporose, rinite, asma, Parkinson e glaucoma, além de fraldas geriátricas para atender idosos no tratamento da incontinência urinária. Já em 2oII, foi lançado a campanha “Saúde Não Tem Preço”. Assim mais 3 medicamentos para o tratamento da asma passaram a ser disponibilizados gratuitamente em oito apresentações: brometo de ipratrópio, diproprionato de beclometasona e sulfato de salbutamol. (BRAGA; ZAIRE, 2008).

Através do projeto são oferecidos medicamentos gratuitos para hipertensão, diabetes e asma, também são oferecidos desconto de 90\%, para farmácias e drogarias cadastradas, em remédios para colesterol alto, Parkinson, rinite, osteoporose e glaucoma. Para diminuir o preço alto de remédios, reduzir a pressão dos gastos com a saúde, assim comprando medicamentos em estabelecimentos credenciados ao projeto, do governo federal. (ZAIRE, 2008).

O cidadão apresenta o cadastro de pessoa física $(\mathrm{CPF})$ e a receita médica ao farmacêutico, importante que o documento esteja carimbado pelo médico e tenha validade de até i8o dias. Após o profissional irá consultar no sistema do Farmácia Popular para verificar a disponibilidade do medicamento, logo, solicitaria a aprovação do desconto de até 90\%. No fim, o consumidor arcará somente com ıo\% do valor do 
remédio e o governo pagará o restante. Esses medicamentos afetam milhões de pessoas pelo país, portanto ganham vantagem nesses remédios subsidiados. (PINTO; COSTA; OSORIO-DE-CASTRO, 2011; SILVA; CAETANO, 2016).

Os estabelecimentos também são beneficiadas com o programa, muitas têm o faturamento atrelado ao sistema de coparticipação, assim o governo paga praticamente tudo adquirido pelo consumidor. Porém, nem tudo é o que parece e no Brasil não seria diferente, no projeto à lacunas abertas, violações e corrupção sendo investigadas. Que intrigam e deixam a população indignada. Houve denuncias feitas no interior de São Paulo pelo Ministério Público Federal em Marília, para 27 empresários por fraude e roubo no programa Farmácia Popular do Brasil. (PINTO; 2016).

Segundo as acusações, donos de drogarias privadas recebiam valores indevidos após simulações de vendas de remédios no sistema, nomes de pessoas já falecidas eram usados. Essas supostas transições ocorreram entre 2010 e 2014 por todo Brasil. A justiça detectou as fraudes, pois, nenhum empresário apresentou as notas fiscais da aquisição dos medicamentos, nem receitas médicas que tivessem relação com à operação. (COSTA; OSORIO-DE-CASTRO, 20II; SILVA; CAETANO, 2016).

Caso segue em investigação pela procuradoria. Outro problema surgiu, em março de 2018 o Ministério da Saúde anunciou que atualizaria os valores de medicamentos referenciados para o tratamento de hipertensão arterial, diabetes mellitus e asma, fornecido pelo programa, e assim reduzindo o repasse em torno de $\mathrm{R} \$ 800$ milhões. Outras duas reduções já haviam sido feitas desde 2009, a iniciativa do governo causou desconforto e preocupação ao setor varejista farmacêutico. (PINTO; COSTA; OSORIO-DE-CASTRO, 20Ir; SILVA; CAETANO, 2016).

Os sócios que representam o setor se manifestaram publicamente com repulsa e chegaram a dizer que essa atitude seria uma ameaça à continuidade do programa, pois farmácias e drogarias receberiam menos em vista os custos fixos dos estabelecimentos, que a cada dia se expandem mais. No fim, ao todo 13 medicamentos sofreram com a redução no repasse, 4 se mantiveram inalterados e 9 reajustados. $\mathrm{Na}$ realidade, isso significa que os estabelecimentos credenciados passaram a receber menos pelos medicamentos. (SILVA; CAETANO, 2016). 
Porém, continua sendo vantajoso participar do programa, que tem como objetivo atrair consumidores de medicamentos para dentro do ponto de venda. Então, o projeto atrai potenciais usuários. Na percepção de especialistas, o ajuste é necessário, já imaginando um futuro colapso no programa. Disseram que até pouco tempo atrás imaginavam que haveria o encerramento do programa, mas viram que o governo tentava se adequar para dar continuidade e beneficiar a população que precisa dos remédios e que, infelizmente, não podem arcar com o valor original. Mesmo que o Programa Popular se resuma à distribuição e dispensação de medicamentos subsidiados pelo governo, é valido lembrar que a Assistência Farmacêutica (AF) não se baseia somente a isso. (SILVA; CAETANO, 2016)

Um fundamental e relevante componente nesse programa é a Atenção Farmacêutica, que orienta sobre o uso racional dos remédios, o acompanhamento e revisão farmacoterapêutico, a educação em saúde, a atenção primária em saúde, entre outras atividades exercidas, que podem/poderiam desonerar o custo do Governo Federal com a saúde pública. (SILVA; CAETANO, 2016).

O programa efetivo no município de Nova Iguaçu, é uma atividade que hoje age com a participação dos farmacêuticos, um forte pilar da farmácia clínica no país. Assim se têm 4 possibilidades que poderiam impactar a profissão farmacêutica no Brasil e região, e qualidade de vida dos cidadãos brasileiros:

I. Para o tratamento de hipertensão arterial, diabetes mellitus e asma, entre outros, a dispensação dos medicamentos poderia ser atrelada ao acompanhamento farmacoterapêutico obrigatório e periódico. Em sua maioria, pacientes crônicos são polimedicados, portanto correm o risco de intoxicação e podendo piorar o quadro clínico de saúde já desenvolvidos. Por isso, a importância da intervenção de farmacêutico clínico que evitaria custos com o SUS e casos de óbitos;

2. Sobre a entrega de medicamentos, podendo ser atrelada à consulta farmacêutica remunerando o farmacêutico pelos serviços clínicos prestados no acompanhamento dos usuários do SUS. O serviço Farmácia Popular de hoje beneficia o paciente com a entrega eficaz do remédio e também do fornecedor, que tem parte de sua receita recebida pelo governo, mas como de costume se esquece de reconhecer o trabalho do profissional que é de extrema importância na relação paciente e o 
medicamento. Infelizmente os farmacêuticos não possuem o reconhecimento merecido;

3. O projeto poderia ampliar a vacinação e humanização dos brasileiros em farmácias, realizadas por farmacêuticos habilitados e qualificados para o procedimento, devidamente remunerados por cada aplicação. O governo economizaria em infraestrutura e com recursos humanos; ganharia o empresário com o valor do produto fornecido e pago pelo governo; ganharia o farmacêutico com essa ampliação de remuneração e maior contato com pacientes na atenção primária à saúde;

4. Na melhor versão, o Programa Popular, por meio da dispensação documentada e organizada via consulta farmacêutica e acompanhamento farmacoterapêutico, podendo ainda ser um grande estudo epidemiológico futuro e contínuo, ajudando com indicadores a melhoria dos padrões e políticas de saúde pública e a ampliação de programas de saúde preventiva a curto e longo prazo.

Muitos "podendo", "poderia" são possibilidades e oportunidades para os farmacêuticos no Programa Farmácia Popular atuando no município de Nova Iguaçu. O objetivo ainda está na iniciativa e na vontade política das entidades líderes da classe, que podem acontecer mediante manifesto dos farmacêuticos do município. (MATTOS, 2015; SILVA; CAETANO, 2016).

\section{DISCURSÃO}

O farmacêutico se caracteriza pela prática de poder interagir diretamente com o cidadão para entender $e$ atender suas necessidades relacionadas aos remédios e o acampamento disciplinar, com o desenvolvimento de atividades clínicas, orientar de forma consciente quanto o uso racional de medicamentos com a obtenção de resultados claros e mensuráveis para a solução dos problemas de saúde do paciente e da comunidade farmacêutica. Nosso país vem intensificando um movimento de intensa reestruturação na área dos remédios que se baseiam no sistema de saúde. Na pesquisa abordada, é importante citar que após o colapso da pandemia, COVID-I9, o Ministério da Saúde divulgou que estaria sem verbas para pagar as despesas com hemoderivados (produtos amplamente usados em procedimentos como transfusão de sangue em caso de hemorragia, em tratamento de hepatite B ou por hemofílicos), assistência médica a 
indígenas, médicos na saúde primárias, bolsas de residentes e abastecimento da Farmácia Popular. A situação levou o Ministério da Economia a liberar recursos para evitar paralisação. E o profissional segue sem ter o merecido reconhecimento em meio a tantas dificuldades. (MACHADO; BAPTISTA; NOGUEIRA, 20II).

\section{CONCLUSÃO}

Na pesquisa foi abordada a atuação do farmacêutico no município de Nova Iguaçu e no Brasil, nas quais as atividades precisam estar centradas não apenas no medicamento, mas no paciente garantindo a utilização de forma adequada, e o suporte a logística necessária à prescrição e dispensação do medicamento, como: participação no planejamento, regularidade do abastecimento (aquisição, armazenamento, distribuição e rastreamento). Ainda há muito a se fazer nessa área, o acesso a medicamentos ainda é uma questão desafiadora para a $\mathrm{AF}$, onde um cenário de dificuldades e barreiras é a realidade do atual governo. Além de não serem reconhecidos e remunerados de forma justa e merecida. O município de Nova Iguaçu poderá entrar em escassez por falta de sensibilidade e qualidade com os farmacêuticos e usuários do programa, podendo assim, acontecer mais fechamentos de estabelecimentos por uma nova redução de repasse futura.

\section{REFERÊNCIAS BIBLIOGRÁFICAS}

AMARAL, S. M. S.; BLATT, C. R. Municipal consortia for medicine procurement: impact on the stock-out and budget. Revista de Saúde Pública, São Paulo, v. 45, n. 4, p. 799-801, 2011.

ARRETCHE, M. Federalismo e políticas sociais no Brasil: problemas de coordenação e autonomia. São Paulo em Perspectiva, São Paulo, v. I8, n. 2, p. 17-26, 2004.

BARDIN, L. Análise de conteúdo. São Paulo: Edições 7o, 201 .

BONOTTO, L. F.; COLET, C. F. Farmácia Popular do Brasil em Ijuí: perfil dos usuários e redução de preços dos produtos. Revista Brasileira de Farmácia, Rio de Janeiro, v. 94, n. I, p. 41-48, 2013. 
BRASIL. Ministério da Saúde. Portaria MS/GM no I.214, de 13 de junho de 2012. Institui o Programa Nacional de Qualificação da Assistência Farmacêutica no âmbito do Sistema Único de Saúde (QUALIFAR-SUS). Diário Oficial da União, Brasília, DF, I4 jun. 2012. Disponível em: <Disponível em: https://bit.ly/2Q1Oldc >. Acesso em: 3 jan. 2019.

BRASIL. Ministério da Saúde. Secretaria de Ciência, Tecnologia e Insumos Estratégicos. Componente populacional: resultados. Brasília, DF, 2016.

CARRARO, W. B. W. H. Desenvolvimento econômico do Brasil e o programa Aqui Tem Farmácia Popular: limitantes e potencialidades. 2014. Tese (Doutorado em Economia) - Universidade Federal do Rio Grande do Sul, Porto Alegre, 2014.

CONASS - CONSELHO NACIONAL DE SECRETARIAS ESTADUAIS DE SAÚDE. Apresentação das dificuldades enfrentadas pelas Secretarias Estaduais de Saúde na aquisição de medicamentos. Brasília, DF, 2014. v. 15. (Série Nota Técnica).

Disponível em: 〈Disponível em: https://bit.ly/2AuNkuo >. Acesso em: 3 jan. 2019. »https://bit.ly/2AuNkuo

COSTA, K. S.; NASCIMENTO JÚNIOR, J. M. Hórus: inovação tecnológica na assistência farmacêutica no sistema único de saúde. Revista de Saúde Pública, São Paulo, v. 46, p. 91-99, 2012. Suplemento I.

FALEIROS, D. R.; SILVA, G. D. Gestão racional da assistência farmacêutica: Farmácia de Minas. In: OSORIO-DE-CASTRO, C. G. S. et al. (Ed.). Assistência farmacêutica: gestão e prática para profissionais da saúde. Rio de Janeiro: Fiocruz, 2014. p. $69-78$.

FERRAES, A. M. B.; CORDONI JÚNIOR, L. Consórcio de medicamentos no Paraná: análise de cobertura e custos. Revista de Administração Pública, Rio de Janeiro, v. 4I, n. 3, p. 475-486, 2007. 
FONSECA, E. M.; COSTA, N. R. Federalism, the economic-industrial health care complex and high-cost pharmaceutical assistance in Brazil. Ciência \& Saúde Coletiva, Rio de Janeiro, v. 20, n. 4, p. 1165-1176, 2015.

FRENKEL, J. Medicamentos: políticas de acesso, segmentação da demanda e progresso técnico. In: BUSS, P. M.; CARVALHEIRO, J. R.; CASAS, C. P. R. (Org.). Medicamentos no Brasil: inovação e acesso. Rio de Janeiro: Fiocruz , 2008. p. 167-197. GUIMARÃES, L.; GIOVANELLA, L. Entre a cooperação e a competição: percursos da descentralização do setor saúde no Brasil. Revista Panamericana de Salud Pública, Washington, DC, v. 16, n. 4, p. 283-288, 2004.

KORNIS, G. E. M.; BRAGA, M. H.; ZAIRE, C. E. F. Os marcos legais das políticas de medicamentos no Brasil contemporâneo (1990-2006). Revista de APS, Juiz de Fora, v. II, n. I, p. 85-99, 2008.

MACHADO, C. V.; BAPTISTA, T. W. F.; NOGUEIRA, C. O. Políticas de saúde no Brasil nos anos 2000: a agenda federal de prioridades. Cadernos de Saúde Pública, Rio de Janeiro, v. 27, n. 3, p. 521-532, 2011.

MACHADO, C. V. et al. Federal funding of health policy in Brazil: trends and challenges. Cadernos de Saúde Pública, Rio de Janeiro, v. 30, n. I, p. 187-200, 2014.

MATTOS, L. V. Assistência farmacêutica na atenção básica e Programa Farmácia Popular do Brasil: uma análise crítica das políticas de provisão de medicamentos no Brasil. 2015. Dissertação (Mestrado em Saúde Pública) - Escola Nacional de Saúde Pública Sergio Arouca, Rio de Janeiro, 2015.

MENDES, L. V. et al. Disponibilidade de medicamentos nas unidades básicas de saúde e fatores relacionados: uma abordagem transversal. Saúde em Debate, Rio de Janeiro, v. 38, p. I09-123, 2014. Número especial. 
OLIVEIRA, M. A. et al. Access to medicines for chronic diseases in Brazil: a multidimensional approach. Revista de Saúde Pública, São Paulo, v. 50, 2016. Suplemento 2.

OPAS - ORGANIZAÇÃO PAN-AMERICANA DE SAÚdE. Avaliação da assistência farmacêutica no Brasil: estrutura, processo e resultados. Brasília, DF, 2005. (Série Medicamentos e Outros Insumos Essenciais para a Saúde).

PINTO, C. D. B. S.; COSTA, N. R.; OSORIO-DE-CASTRO, C. G. S. Quem acessa o Programa Farmácia Popular do Brasil? Aspectos do fornecimento público de medicamentos. Ciência \& Saúde Coletiva, Rio de Janeiro, v. 16, n. 6, p. 2963-2973, 2011.

PINTO, C. D. B. S. et al. Medicine prices and availability in the Brazilian popular pharmacy program. Revista de Saúde Pública, São Paulo, v. 44, n. 4, p. 6II-6ı, 2010.

SILVA, R. M.; CAETANO, R. Costs of public pharmaceutical services in Rio de Janeiro compared to Farmácia Popular Program. Revista de Saúde Pública, São Paulo, v. 50, 2016.

VIEIRA, F. S. Qualificação dos serviços farmacêuticos no Brasil: aspectos inconclusos da agenda do Sistema Único de Saúde. Revista Panamericana de Salud Pública, Washington, DC, v. 24, n. 2, p. 91-100, 2008. 